\title{
Low kV High Resolution Scanning Electron Microscopy Study of Si Nanowire Surfaces
}

C. Alfonso ${ }^{1}$, L. Roussel ${ }^{1}$, A. Charaï ${ }^{1}$, C. Dominici ${ }^{2}$, A. P. C. Campos ${ }^{2}$, L.Y. Han ${ }^{3}$, F. Zhou $^{3}$

1. Aix-Marseille Université - CNRS, IM2NP, Faculté des Sciences, Campus de St Jérôme, F-13397 Marseille, France

2. Aix-Marseille Université, CP2M, Faculté des Sciences, Campus de St Jérôme, F-13397 Marseille, France

3. Carl Zeiss Microscopy, GmbH, Carl-Zeiss-Straße 22, 73447 Oberkochen, Germany

The characterization of small objects like nanometric gold nanocrystals on nanowire (NW) surface allowed us to highlight the improvements of new imaging detectors in SEM. We have employed different detectors for both conventional imaging signals (SE and BSE), coupled with analytical techniques. In previous work [3], saw-tooth faceting and non-homogeneous gold repartition have been characterized by coupling Scanning Transmission Electron Microscopy - High Angle Annular Dark Field (STEM-HAADF), electron tomography and Energy-dispersive X-ray spectroscopy (EDS).

This work deals with surface faceting and post-growth repartition of the gold used as catalyst during Molecular Beam Epitaxy (MBE) growth of silicon NWs. Indeed, because of the high surface/volume ratio, NWs surface investigation is fundamental to explain NWs properties and to functionalize them in order to develop new applications as, for example, the detection of chemical or biological species [1, 2].

In this paper, we show that new FESEM at low $\mathrm{kV}$ can provide very similar information not only about the surface morphology but also about its composition with high resolution.

Fig 1 and 2 show the first results obtained with the Zeiss GeminiSEM 500 ultra-high resolution FESEM, just installed in CP2M Aix-Marseille University. For imaging, this system is completely equipped with In-lens SE and the Energy Selective Backscattered detector (EsB), annular-BF-DF-HAADF-STEM, five quadrant multi-mode solid state BSE, variable pressure (VP) SE and VPBSE detectors. For the chemical and crystallographic analyses, EDS, WDS, and EBSD accessories are additionally installed on this microscope achieving the full imaging and analytical capabilities.

Fig 1 shows clearly the transition to six to twelve faces from the bottom to the top of the NW. Moreover, the saw-tooth faceting of one over two faces is also visible with a high resolution. This finer faceting is generally difficult to resolve with common SEMs. In Fig 2, because of filtered in-lens BSE imaging at low $\mathrm{kV}$, a strong chemical contrast is achieved. Gold particles appear bright. It is now possible not only to distinguish the catalyst on the NW but also to resolve nanometric gold nanocrystals on the different surfaces and to analyze their repartition.

Such a resolution, coupled to chemical sensitivity, was only possible, up to now, with STEM-HAADF. But, SEM analysis has the advantage of not having any previous preparation such as nanowire dispersion and/or thin foil preparation. Moreover, SEM provides a larger field of view and a better statistics in NW characterization. The low voltage acquisition was adequate for our surface studies since it presents less delocalisation. 
More studies are under development to compare analytical performance of this new SEM with the TEM one. At this stage, we believe this new equipment as an indispensable tool to investigate objects at the mesoscopic scale. There is a huge application potential to investigate nano-objects at different scales without destructive manipulation.

References:

[1] J. Wang, Biomolecule-functionalized nanowires: From nanosensors to nanocarriers, Chem. Phys. Chem. 10 (11) (2009) 1748-1755.

[2] G-S. Park et al., Full Surface Embedding of Gold Clusters on Silicon Nanowires for Efficient Capture and Photothermal Therapy of Circulating Tumor Cells, Nano Lett. 12, (2012) 1638-1642

[3] T. David et al., Gold coverage and faceting of MBE grown silicon nanowires, J. Cryst. Growth 383 (2013) 151-157

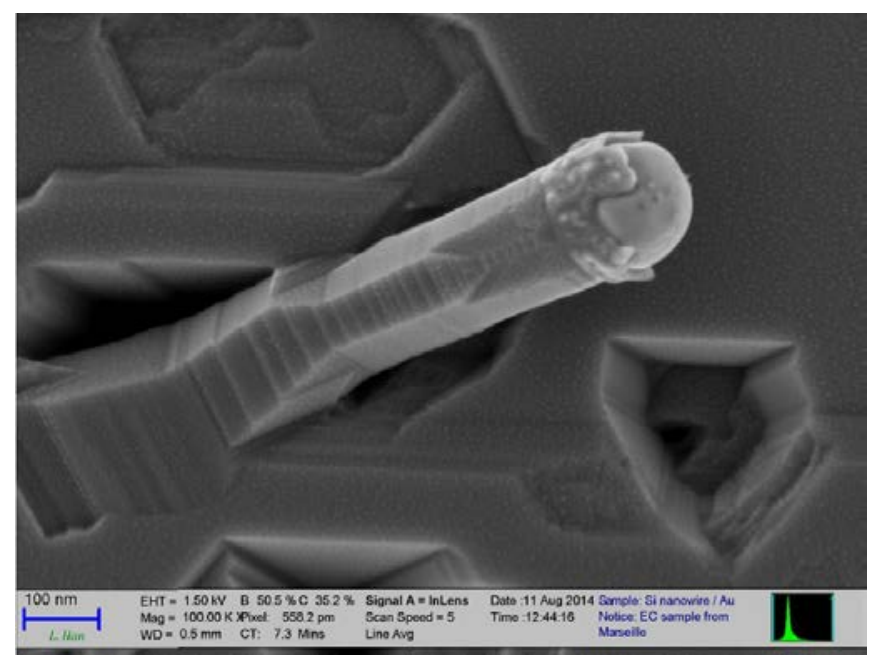

Figure 1. In-lens SE detector image of a Si NW clearly showing different facets and its fine structure on the surface. The surface sensitive imaging at $1.5 \mathrm{kV}$ reveals highly resolved crystalline facets on the Si NW.

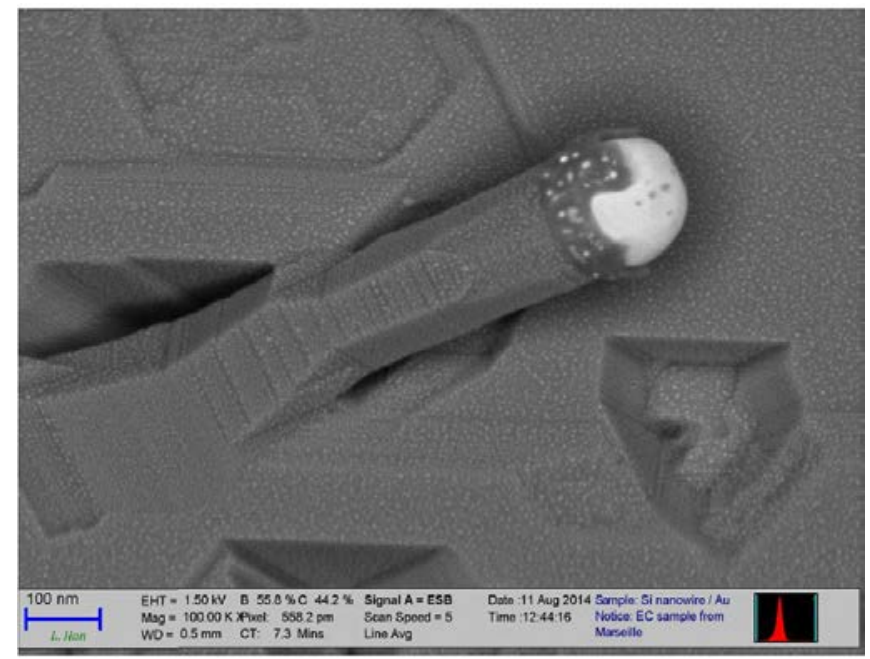

Figure 2. EsB detector image of a Si NW, unveiling a gold catalytic particle on top of the pillar and small gold nanoparticles on the surface. High resolution compositional imaging is only possible using the filtered in-lens BSE imaging at low kV. Both pictures shown in Fig.1 and 2 are acquired in parallel and at low $\mathrm{kV}$. 